\title{
Craig Interpolation in the Presence of Unreliable Connectives
}

\author{
J. Rasga C. Sernadas A. Sernadas \\ Dep. Matemática, Instituto Superior Técnico, Universidade de Lisboa, Portugal \\ and \\ SQIG, Instituto de Telecomunicações, Lisboa, Portugal \\ \{joao.rasga,cristina.sernadas,amilcar.sernadas\}@tecnico.ulisboa.pt
}

March 24, 2014

\begin{abstract}
Arrow and turnstile interpolations are investigated in UCL (introduced in [32]), a logic that is a complete extension of classical propositional logic for reasoning about connectives that only behave as expected with a given probability. Arrow interpolation is shown to hold in general and turnstile interpolation is established under some provisos.
\end{abstract}

Mathematics Subject Classification (2010). 03B48; 68T37; 03B60; 03B70

Keywords. Probabilistic logic, unreliable connectives, arrow and turnstile interpolations

\section{Introduction}

Interpolation was introduced by Craig for first-order logic in a proof-theoretic context $[11,12]$ and since then has been investigated for many different logics. Namely, interpolation was also addressed in intuitionistic, modal and positive logics [19], in provability logic [3], interpretability and hybrid logics [2, 1], as well as in substructural logics $[22,26]$. Moreover, it was further extended to manysorted logics [17]. Some negative examples are also reported in the literature, concerning, in particular, modal and relevant logics [31, 37].

Two forms of Craig interpolation are extensively discussed in the literature. Arrow interpolation assumes that the logic has implication and states that if $\varphi \supset \psi$ is a theorem then there is a formula $\theta$ using only common symbols of $\varphi$ and $\psi$ such that $\varphi \supset \theta$ and $\theta \supset \psi$ are theorems. Turnstile interpolation does not require the presence of implication in the logic and states that if $\psi$ is derived from $\varphi$ then there is a set of formulas $\Theta$ using only common symbols of $\varphi$ and $\psi$ such that each formula of $\Theta$ is derived from $\varphi$, and $\psi$ is derived from $\Theta$. Arrow and turnstile interpolation notions can be related when the logic has the 
metatheorems of deduction and modus ponens. Other forms of interpolation have also been considered in the literature like uniform interpolation [15] and Maehara interpolation [25, 13], among others.

Craig interpolation has been addressed using proof theoretic techniques as well as using (non constructive) model theoretic abstractions like amalgamation. Some fundamental work on the proof theoretic front was addressed in $[7,8]$. Concerning the model theoretic line of research we should point out the results included in $[19,14,18]$ mainly for modal-like logics and algebraic logics. In [16] a general framework for analyzing interpolation is proposed in the context of institutions.

Preservation of interpolation has been investigated in the context of combination of logics like fusion [23], fibring [10] and meet combination [33, 34]. Furthermore, interpolation can be preserved by back and forth translations [9]. Usually these results provide sufficient conditions for the preservation of interpolation. Several results on this front concern turnstile interpolation since the logics at hand may not have an implication connective or the component logics may have different kinds of implication.

Besides being an important logical property by itself, Craig interpolation has applications in definability [4], projective classes [24] and automated reasoning [6]. Moreover, Craig interpolation has been applied in modular specification [5], model checking of software applications [28, 20, 29] and in logics with interacting agents [21].

Despite recent work on interpolation for logics with non-deterministic features, namely in fuzzy logics [30] and stochastic satisfiability [36], it seems that there is still scope for work in this area. Herein we discuss interpolation for a logic called UCL, introduced in [32], extending propositional logic in a conservative way with some connectives having a probabilistic behavior. More precisely, each occurrence of an unreliable connective $\widetilde{c}$ in a formula behaves as $c$ with probability $\nu$ and as $\bar{c}$ (negated $c$ ) with probability $1-\nu$. In this setting, we discuss both arrow and turnstile interpolations as well as their relationship. The results obtained are very different from those in the context of propositional logic since in UCL not all instances of propositional tautologies are valid, modus ponens does not hold in general and the metatheorems of deduction and modus ponens hold with provisos.

In Section 2, we provide a brief overview of UCL including syntax, Hilbert calculus and semantics. In Section 3, we present the relevant interpolation concepts. We start by considering in Subsection 3.1 pure propositional based logics and analyze the relationship between turnstile and arrow interpolations when the metatheorems of deduction and modus ponens are available. Then, in Subsection 3.2, we focus on the appropriate notions for capturing interpolation in the context of $U C L$ and again relate arrow and turnstile interpolations. In Section 4, we show that UCL has arrow interpolation and in Section 5 we prove that UCL enjoys turnstile interpolation with some provisos. Finally, in Section 6 we draw some conclusions and outline future work. 


\section{An Overview of UCL}

The logic of unreliable connectives UCL, introduced in [32], is a conservative sound and complete extension of propositional logic that includes connectives that only behave properly with a given probability. For example, $\widetilde{\neg}$ is an unreliable connective that when applied to a propositional formula $\varphi$ produces the formula $\neg \varphi$ that behaves as $\neg \varphi$ with probability $\nu$ and behaves as $\bar{\varphi} \varphi$ with probability $1-\nu$.

We provide here only a brief overview of the main features of UCL, namely syntax, semantics and Hilbert calculus.

Let $\Sigma$ be the signature for the propositional logic PL containing the propositional constants $\mathrm{tt}$ (verum) and ff (falsum) plus the propositional connectives $\neg$ (negation), $\wedge$ (conjunction),$\vee$ (disjunction),$\supset$ (implication) and $\equiv$ (equiv-

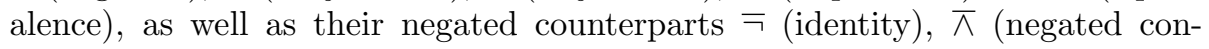
junction), $\bar{\nabla}$ (negated disjunction), $\bar{\supset}$ (negated implication), and $\equiv$ (negated equivalence). Throughout this paper, $L(X)$ is the set of propositional formulas over $\Sigma$ using a given set $X$ of propositional variables.

The signature of $\mathrm{UCL}$ is the triple $\left(\Sigma^{\mathrm{uc}}, \nu, \mu\right)$ where:

- $\Sigma^{\mathrm{uc}}$ contains $\Sigma$ and the following unreliable connectives:

$$
\widetilde{\neg}, \widetilde{\bar{ᄀ}}, \widetilde{\wedge}, \widetilde{\bar{\wedge}}, \widetilde{\nabla}, \widetilde{\nabla}, \widetilde{\supset}, \widetilde{\bar{\supset}}, \cong \text { and } \cong
$$

- both $\nu$ and $\mu$ are symbols used for denoting probabilities.

Each unreliable connective is assumed to behave properly with probability $\nu$. A formula is assumed to behave properly with a probability not less than $\mu$.

Observe that we could have introduced most of the unreliable connectives as abbreviations of the unreliable connective $\widetilde{\bar{ᄀ}}$, see [32].

By $\widetilde{\Sigma}$ one means the subsignature composed of the unreliable connectives in $\Sigma^{\text {uc }}$. Thus, $\Sigma^{\text {uc }}=\Sigma \cup \widetilde{\Sigma}$. Moreover, for each $n \in \mathbb{N}, \Sigma_{n}, \Sigma_{n}^{\text {uc }}$ and $\widetilde{\Sigma}_{n}$ are the set

of $n$-ary constructors in $\Sigma$, $\Sigma^{\text {uc }}$ and $\widetilde{\Sigma}$, respectively. Observe that $\widetilde{\Sigma}_{0}=\emptyset$ and $\Sigma_{n}^{\mathrm{uc}}=\emptyset$ for $n>2$.

Given a PL formula $\varphi$ and a UCL formula $\psi$, one writes

$$
\varphi \sqsubseteq \psi
$$

for saying that $\varphi$ is a possible outcome of $\psi$. This outcome relation is inductively defined as expected:

- $\varphi \sqsubseteq \varphi$ provided that $\varphi$ is a PL formula;

- $c\left(\varphi_{1}, \ldots, \varphi_{n}\right) \sqsubseteq c\left(\psi_{1}, \ldots, \psi_{n}\right)$ provided that $n \geq 1, c \in \Sigma_{n}$ and $\varphi_{i} \sqsubseteq \psi_{i}$ for $i=1, \ldots, n$;

- $c\left(\varphi_{1}, \ldots, \varphi_{n}\right) \sqsubseteq \widetilde{c}\left(\psi_{1}, \ldots, \psi_{n}\right)$ provided that $\widetilde{c} \in \widetilde{\Sigma}_{n}$, and $\varphi_{i} \sqsubseteq \psi_{i}$ for $i=1, \ldots, n$; 
- $\bar{c}\left(\varphi_{1}, \ldots, \varphi_{n}\right) \sqsubseteq \widetilde{c}\left(\psi_{1}, \ldots, \psi_{n}\right)$ provided that $\widetilde{c} \in \widetilde{\Sigma}_{n}$, and $\varphi_{i} \sqsubseteq \psi_{i}$ for $i=1, \ldots, n$.

For each such $\psi$,

$$
\Omega_{\psi}
$$

is the set $\{\varphi: \varphi \sqsubseteq \psi\}$ of all possible outcomes of $\psi$. Clearly, $\Omega_{\varphi}=\{\varphi\}$ for each PL formula $\varphi$.

Terms exist in UCL for denoting probabilities. The signature of the firstorder theory of ordered real closed fields ORCF (shown to be decidable in [35]) is used for writing such terms. Recall that the signature of ORCF contains the constants 0 and 1 , the unary function symbol -, the binary function symbols + and $\times$, and the binary predicate symbols $=$ and $<$. As usual, one may write $t_{1} \leq t_{2}$ for $\left(t_{1}<t_{2}\right) \vee\left(t_{1}=t_{2}\right)$ and $t_{1} t_{2}$ for $t_{1} \times t_{2}$.

In UCL, by a term one means a univariate polynomial in ORCF, using $\nu$ as the variable. Symbol $\mu$ is also taken as a variable in the context of ORCF but it is not used in UCL terms.

Three kinds of formulas exist in UCL for reasoning about formulas with unreliable connectives:

- Formulas (possibly) with unreliable connectives ${ }^{1}$ that are formulas built with the symbols in $\Sigma^{\mathrm{uc}}$ and $X$. Throughout this paper, $L^{\mathrm{c}}(X)$ is the set of such formulas, henceforth known as $c$-formulas. For instance, the formula

$$
\left(x_{1} \widetilde{V}\left(\widetilde{\neg} x_{2}\right)\right) \widetilde{\wedge} x_{3}
$$

in $L^{\mathrm{c}}(X)$ has three unreliable connectives, while the formula

$$
\left(x_{1} \vee\left(\neg x_{2}\right)\right) \wedge x_{3}
$$

has no unreliable connectives. The formula $\psi \equiv \varphi$, where $\psi \in L^{\mathrm{C}}(X)$ and $\varphi \in L(X)$, is intended to state that $\psi$ is equivalent to $\varphi$ with probability at least $\mu$.

- Outcome formulas that are of the general form

$$
\Phi \sqsubseteq_{P} \psi
$$

where $\psi \in L^{\mathrm{c}}(X), \Phi \subseteq \Omega_{\psi}$ and $P$ is a term. Such an outcome formula is intended to state that the probability of an outcome of $\psi$ being in $\Phi$ is at least $P$. Throughout this paper, $L^{\circ}(X)$ is the set of outcome formulas. For instance,

$$
\left\{\left(x_{1} \vee\left(\neg x_{2}\right)\right) \wedge x_{3},\left(x_{1} \bar{\nabla}\left(\neg x_{2}\right)\right) \wedge x_{3}\right\} \sqsubseteq_{\nu^{2}}\left(x_{1} \widetilde{\vee}\left(\neg x_{2}\right)\right) \widetilde{\wedge} x_{3}
$$

should be true in any interpretation of UCL because the probability of the outcome $\left(x_{1} \vee\left(\neg x_{2}\right)\right) \wedge x_{3}$ is $\nu^{3}$, the probability of the outcome $\left(x_{1} \nabla\right.$ $\left.\left(\neg x_{2}\right)\right) \wedge x_{3}$ is $(1-\nu) \nu^{2}$, and so their aggregated probability is

$$
\nu^{3}+(1-\nu) \nu^{2}=\nu^{2} .
$$

\footnotetext{
${ }^{1}$ Called circuit fomulas in [32] since they are used there for representing circuits with unreliable gates.
} 
We may use $\varphi_{1}, \ldots, \varphi_{m} \sqsubseteq_{P} \psi$ instead of $\left\{\varphi_{1}, \ldots, \varphi_{m}\right\} \sqsubseteq_{P} \psi$.

- Ambition formulas that are of the general form

$$
\mu \leq P
$$

where $P$ is a term. Such an ambition formula can be used for constraining the envisaged truth probability $\mu$ of the c-formulas at hand. Throughout this paper, $L^{\mathrm{a}}$ is the set of ambition formulas. For example, every interpretation of UCL where the ambition formula

$$
\mu \leq \nu^{2}+(1-\nu)^{2}
$$

holds should make the c-formula

$$
\left(\neg\left(x_{1} \vee x_{2}\right)\right) \equiv\left(\neg\left(x_{1} \widetilde{\vee} x_{2}\right)\right)
$$

true, since the probability of outcome $\neg\left(x_{1} \vee x_{2}\right)$ is $\nu^{2}$, the probability of

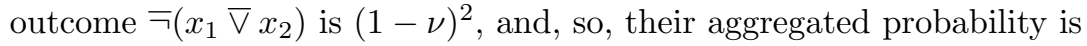

$$
\nu^{2}+(1-\nu)^{2} .
$$

In the sequel, $L^{\mathrm{uc}}(X)$ is the set $L^{\mathrm{c}}(X) \cup L^{\circ}(X) \cup L^{\mathrm{a}}$ of all $\mathrm{UCL}$ formulas. Given $\psi \in L^{\mathrm{C}}(X)$ and $\varphi \in \Omega_{\psi}$, one writes

$$
\mathfrak{P}[\psi \triangleright \varphi]
$$

for the UCL term that provides the probability of outcome $\varphi$ of $\psi$. This term is inductively defined as follows:

- $\mathfrak{P}[\varphi \triangleright \varphi]$ is 1 for each $\varphi \in L(X)$;

- $\mathfrak{P}\left[c\left(\psi_{1}, \ldots, \psi_{n}\right) \triangleright c\left(\varphi_{1}, \ldots, \varphi_{n}\right)\right]$ is $\prod_{i=1}^{n} \mathfrak{P}\left[\psi_{i} \triangleright \varphi_{i}\right]$ for each $n \geq 1, c \in \Sigma_{n}$ and $\varphi_{i} \sqsubseteq \psi_{i}$ for $i=1, \ldots, n$;

- $\mathfrak{P}\left[\widetilde{c}\left(\psi_{1}, \ldots, \psi_{n}\right) \triangleright c\left(\varphi_{1}, \ldots, \varphi_{n}\right)\right]$ is $\nu \prod_{i=1}^{n} \mathfrak{P}\left[\psi_{i} \triangleright \varphi_{i}\right]$ for each $\widetilde{c} \in \widetilde{\Sigma}_{n}$ and $\varphi_{i} \sqsubseteq \psi_{i}$ for $i=1, \ldots, n$;

- $\mathfrak{P}\left[\widetilde{c}\left(\psi_{1}, \ldots, \psi_{n}\right) \triangleright \bar{c}\left(\varphi_{1}, \ldots, \varphi_{n}\right)\right]$ is $(1-\nu) \prod_{i=1}^{n} \mathfrak{P}\left[\psi_{i} \triangleright \varphi_{i}\right]$ for each $\widetilde{c} \in \widetilde{\Sigma}_{n}$ and $\varphi_{i} \sqsubseteq \psi_{i}$ for $i=1, \ldots, n$.

For instance, the term $\mathfrak{P}\left[\neg\left(x_{1} \widetilde{\vee} x_{2}\right) \triangleright \neg\left(x_{1} \bar{\nabla} x_{2}\right)\right]$ is the polynomial $\nu(1-\nu)$.

Regarding semantics, each interpretation of $U C L$ should provide a valuation to the variables in $X$, a model of ORCF and an assignment to the variables $\nu$ and $\mu$. However, the choice of the model of ORCF is immaterial since all such 
models are elementarily equivalent (see Corollary 3.3.16 in [27]) and, so, one can adopt the ordered field $\mathbb{R}$ of the real numbers.

Accordingly, an interpretation in $\mathrm{UCL}$ is a pair

$$
I=(v, \rho)
$$

where $v$ is a propositional valuation and $\rho:\{\mu, \nu\} \rightarrow \mathbb{R}$ is an assignment such that:

$$
\left\{\begin{array}{l}
\frac{1}{2}<\rho(\mu) \leq 1 \\
\frac{1}{2}<\rho(\nu) \leq 1
\end{array}\right.
$$

We now proceed to review the definition of satisfaction in UCL by a given interpretation $I=(v, \rho)$. To this end, one relies on the first-order logic satisfaction $\Vdash^{\text {fo }}$ and on the propositional logic satisfaction $\Vdash$.

Starting with formulas in $L^{\mathrm{c}}(X)$,

$$
I \Vdash^{\text {uc }} \psi
$$

states that

$$
\mathbb{R} \rho \Vdash^{\text {fo }} \mu \leq \sum_{\substack{\varphi \sqsubseteq \psi \\ v \Vdash \varphi}} \mathfrak{P}[\psi \triangleright \varphi] .
$$

That is, the aggregated probability of the outcomes of $\psi$ that are (classically) satisfied by $v$ is at least the value of $\mu$.

Concerning formulas in $L^{\circ}(X)$,

$$
I \Vdash^{\mathrm{uc}} \Phi \sqsubseteq_{P} \psi
$$

states that

$$
\mathbb{R} \rho \Vdash^{\text {fo }} P \leq \sum_{\varphi \in \Phi} \mathfrak{P}[\psi \triangleright \varphi] .
$$

That is, the collection $\Phi$ of possible outcomes of $\psi$ has aggregated probability greater than or equal to the value of $P$.

Finally, concerning formulas in $L^{\text {a }}$,

$$
I \Vdash^{\mathrm{uc}} \mu \leq P
$$

states that

$$
\mathbb{R} \rho \Vdash^{\text {fo }} \mu \leq P \text {. }
$$

That is, the required probability $\rho(\mu)$ for a formula in $L^{\mathrm{c}}(X)$ to behave properly does not exceed the value of $P$.

Satisfaction is extended to mixed sets of formulas in $L^{\circ}(X)$, formulas in $L^{\mathrm{c}}(X)$ and formulas in $L^{\text {a }}$ with no surprises. Given $\Upsilon \subseteq L^{\text {uc }}(X), I \Vdash^{\text {uc }} \Upsilon$ if $I \Vdash^{\text {uc }} v$ for each $v \in \Upsilon$.

Moreover, entailment and validity in UCL are also defined as expected. Given $\{\theta\} \cup \Upsilon \subseteq L^{\text {uc }}(X)$

$$
\Upsilon \vDash^{\mathrm{uc}} \theta
$$


states that $\Upsilon$ entails $\theta$ in the following sense:

$$
I \Vdash^{\text {uc }} \theta \text { whenever } I \Vdash^{\text {uc }} \Upsilon \text {, for every interpretation } I \text {. }
$$

Finally, one writes $\vDash^{\text {uc }} \theta$ for $\emptyset \vDash^{\text {uc }} \theta$ in which case one says that $\theta$ is valid.

The calculus of UCL capitalizes on the decidability of the following problems which are used in some provisos: validity in $\mathrm{PL}$, membership in $L(X)$, emptyness of intersection of two finite sets and theoremhood in ORCF. It contains the following axioms and rules:

- the PL tautologies as axioms:

TAUT $\frac{}{\varphi}$ provided that $\varphi \in L(X)$ and $\vDash \varphi$;

- the modus ponens rule:

$$
\operatorname{MP} \frac{\psi \supset \varphi}{\varphi} \text { provided that } \varphi \in L(X) ;
$$

- the following outcome axioms:

$$
\text { NO } \frac{\emptyset_{0} \psi}{\varphi \sqsubseteq_{\mathfrak{P}[\psi \triangleright \varphi]} \psi} ;
$$

- the following outcome rules:

AO $\frac{\Phi_{i} \sqsubseteq_{P_{i}} \psi \text { for } i=1,2}{\Phi_{1} \cup \Phi_{2} \sqsubseteq_{\left(P_{1}+P_{2}\right)} \psi}$ provided that $\Phi_{1} \cap \Phi_{2}=\emptyset$;

WO $\frac{\Phi \sqsubseteq_{P_{1}} \psi}{\Phi \sqsubseteq_{P_{2}} \psi}$ provided that ${ }^{2}$

$$
\forall \nu\left(\left(\frac{1}{2}<\nu \leq 1\right) \supset P_{2} \leq P_{1}\right) \in \mathrm{ORCF}
$$

- the following ambition rule for each $k \in \mathbb{N}$ :

$$
\begin{aligned}
\text { WA } \frac{\mu \leq P_{i} \text { for } i=1, \ldots, k}{\mu \leq P} \text { provided that }{ }^{3} \\
\qquad \forall \forall \nu\left(\left(\frac{1}{2}<\mu, \nu \leq 1 \wedge \bigwedge_{i=1}^{k} \mu \leq P_{i}\right) \supset \mu \leq P\right) \in \text { ORCF; }
\end{aligned}
$$

- the following unreliable connectives rule for each $k \in \mathbb{N}^{+}:{ }^{4}$

\footnotetext{
${ }^{2}$ Writing $P<z \leq P^{\prime}$ for $P<z \wedge z \leq P^{\prime}$.

${ }^{3}$ Writing $P<z, z^{\prime} \leq P^{\prime}$ for $P<z \leq P^{\prime} \wedge P<z^{\prime} \leq P^{\prime}$.

${ }^{4}$ Given a finite set $\bar{\Phi}$ of PL formulas, we write $\Lambda \bar{\Phi}$ for the conjunction of the formulas in $\Phi$. As usual, this conjunction is tt when $\Phi=\emptyset$.
} 


$$
\begin{gathered}
\bigvee_{i=1}^{k}\left(\bigwedge \Phi_{i}\right) \\
\text { LFT } \frac{\left\{\begin{array}{l}
\Phi_{i} \sqsubseteq_{P_{i}} \psi \\
\mu \leq P_{i}
\end{array} \text { for } i=1, \ldots, k\right.}{\psi} .
\end{gathered}
$$

The reader will wonder why the (propositional) tautologies were taken as axioms (TAUT) but not all their UCL instances. In fact, such instances are not valid in general in UCL. For example, the unreliable instance $(\neg x) \supset(\neg x)$ of the tautology $x \supset x$ is not valid.

The reader will also wonder if MP is sound when the conclusion is a formula in $L^{\mathrm{c}}(X)$. It is not always so since, for example,

$$
\widetilde{\neg} x_{1},\left(\neg x_{1}\right) \supset\left(\neg x_{2}\right) \not \not^{\text {uc }} \neg x_{2} .
$$

Most of the other axioms and rules are self-explanatory. But the lifting rule (LFT) does require some motivation. Borrowing an example from [32], assume that we want to infer that

$$
\left(\left(\widetilde{\neg} x_{1}\right) \wedge x_{2}\right) \supset\left(\widetilde{\neg} x_{1}\right)
$$

holds with probability not smaller than $\mu$. We can do so provided that we constrain our ambition to $\mu$ being not greater than $\nu^{2}-\nu+1$. Indeed, it is easy to verify that

$$
\left(\bigwedge \Phi_{1}\right) \vee\left(\bigwedge \Phi_{2}\right)
$$

is a tautology as long as

$$
\left\{\begin{array}{l}
\Phi_{1}=\left\{\varphi_{1}, \varphi_{3}, \varphi_{4}\right\} \\
\Phi_{2}=\left\{\varphi_{1}, \varphi_{2}, \varphi_{4}\right\}
\end{array}\right.
$$

where

$$
\left\{\begin{array}{l}
\varphi_{1} \text { is }\left(\left(\neg x_{1}\right) \wedge x_{2}\right) \supset\left(\neg x_{1}\right) \\
\varphi_{2} \text { is }\left((\bar{\neg}) \wedge x_{2}\right) \supset\left(\neg x_{1}\right) \\
\varphi_{3} \text { is }\left(\left(\neg x_{1}\right) \wedge x_{2}\right) \supset\left(\neg x_{1}\right) \\
\varphi_{4} \text { is }\left(\left(\neg x_{1}\right) \wedge x_{2}\right) \supset\left(\neg x_{1}\right) .
\end{array}\right.
$$

Furthermore, it is straightforward to verify that both

$$
\Phi_{1} \sqsubseteq_{\nu^{2}-\nu+1}\left(\left(\neg x_{1}\right) \wedge x_{2}\right) \supset\left(\neg x_{1}\right)
$$

and

$$
\Phi_{2} \sqsubseteq_{\nu^{2}-\nu+1}\left(\left(\neg x_{1}\right) \wedge x_{2}\right) \supset\left(\neg x_{1}\right)
$$

hold. Thus, assuming that

$$
\mu \leq \nu^{2}-\nu+1
$$


the LFT rule allows us to conclude that

$$
\left(\left(\neg x_{1}\right) \wedge x_{2}\right) \supset\left(\neg x_{1}\right)
$$

holds with probability not smaller than $\mu$. In fact, for each $i=1,2$, we were able to collect in $\Phi_{i}$ outcomes of $\left(\left(\neg x_{1}\right) \wedge x_{2}\right) \supset\left(\neg x_{1}\right)$ with aggregated probability not smaller than the ambitioned upper bound for $\mu$.

In the sequel we will often use the derived rule

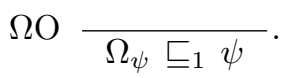

Derivability and theoremhood in this calculus are defined as usual. Given $\{\theta\} \cup \Upsilon \subseteq L^{\mathrm{uc}}(X)$,

$$
\Upsilon \vdash^{\mathrm{uc}} \theta
$$

states that $\theta$ is derivable from $\Upsilon$, that is, states that there is a derivation sequence for obtaining $\theta$ from the elements of $\Upsilon$ (as hypotheses) and the axioms, using the rules of the calculus. Furthermore, when $\emptyset \vdash^{\text {uc }} \theta$, written $\vdash^{\text {uc }} \theta$, one says that $\theta$ is a theorem of UCL.

\section{Interpolation Notions}

We start by very briefly overviewing Craig interpolation in the general context of pure propositional based logics before discussing Craig interpolation in UCL.

\subsection{Craig Interpolation in Pure Propositional Based Log- ics}

Pure propositional based logics are conservative extensions of propositional logic with just one kind of formulas, generated from a set $X$ of propositional variables along with the propositional and possibly additional connectives. For instance, modal logics are in this category. Two notions of Craig interpolation, turnstile interpolation and arrow interpolation, are addressed herein. We denote by $\operatorname{var}(\eta)$, where $\eta$ is a formula, the set of all propositional variables that occur in $\eta$.

A logic is said to enjoy the turnstile interpolation property for a set of formulas $\Delta$ and a formula $\eta$ with $\Delta \vdash \eta$, if

there is $\Theta$ such that

(1) $\operatorname{var}(\Theta) \subseteq \operatorname{var}(\Delta) \cap \operatorname{var}(\eta)$

(2) $\Delta \vdash \theta$ for each $\theta \in \Theta$ and $\Theta \vdash \eta$

whenever $\operatorname{var}(\Delta) \cap \operatorname{var}(\eta) \neq \emptyset$,

otherwise either $\Delta \vdash \mathrm{ff}$ or $\vdash \eta$.

When $\operatorname{var}(\Delta) \cap \operatorname{var}(\eta) \neq \emptyset$, such a $\Theta$ is said to be a turnstile interpolant for $\Delta$ and $\eta$. A logic is said to enjoy the turnstile interpolation property whenever it enjoys turnstile interpolation for every $\Delta$ and $\eta$ such that $\Delta \vdash \eta$. 
A logic is said to enjoy the arrow interpolation property for formulas $\eta$ and $\eta^{\prime}$ with $\vdash \eta \supset \eta^{\prime}$, if

$$
\begin{aligned}
& \text { there is } \theta \text { such that } \\
& \qquad \begin{array}{l}
\text { (1) } \operatorname{var}(\theta) \subseteq \operatorname{var}(\eta) \cap \operatorname{var}\left(\eta^{\prime}\right) \\
\quad(2) \vdash \eta \supset \theta \text { and } \vdash \theta \supset \eta^{\prime}
\end{array} \\
& \text { whenever } \operatorname{var}(\eta) \cap \operatorname{var}\left(\eta^{\prime}\right) \neq \emptyset \text {, } \\
& \text { otherwise either } \vdash \eta \supset \text { ff or } \vdash \eta^{\prime} .
\end{aligned}
$$

When $\operatorname{var}(\eta) \cap \operatorname{var}\left(\eta^{\prime}\right) \neq \emptyset$, such a $\theta$ is said to be an arrow interpolant for $\eta$ and $\eta^{\prime}$. A logic is said to enjoy the arrow interpolation property whenever it enjoys arrow interpolation for every $\eta$ and $\eta^{\prime}$ such that $\vdash \eta \supset \eta^{\prime}$.

Observe that the above definitions follow the original one proposed by Craig (see [11]) and are more restrictive than other forms of interpolation not imposing any conditions when the set of common propositional variables is empty. For example, the minimal normal modal logic $\mathrm{K}$ does not enjoy the Craig interpolation property as defined above.

We start by showing that a turnstile interpolant can be reduced to a single interpolant.

Proposition 3.1 Consider a pure propositional based logic, a set of formulas $\Delta$ and a formula $\eta$ such that $\Delta \vdash \eta$ and $\operatorname{var}(\Delta) \cap \operatorname{var}(\eta) \neq \emptyset$. Then $\Delta$ and $\eta$ have a turnstile interpolant if and only if they have a singleton turnstile interpolant.

Proof: $(\rightarrow)$ Assume that the set of formulas $\Theta$ is a turnstile interpolant for $\Delta$ and $\eta$. Let $\Theta^{\prime}$ be a finite subset of $\Theta$ such that $\Theta^{\prime} \vdash \eta$. Then

- $\operatorname{var}\left(\bigwedge \Theta^{\prime}\right) \subseteq \operatorname{var}(\Delta) \cap \operatorname{var}(\eta)$

- $\Delta \vdash \wedge \Theta^{\prime}$;

- $\wedge \Theta^{\prime} \vdash \eta$.

Thus, $\left\{\wedge \Theta^{\prime}\right\}$ is a turnstile interpolant for $\Delta$ and $\eta$.

$(\leftarrow)$ Immediate.

We now discuss the relationship between the interpolation properties and the metatheorems of deduction (MTD) and of modus ponens (MTMP).

We say that a pure propositional based logic has the MTMP whenever: if $\Delta \vdash \eta \supset \eta^{\prime}$ then $\Delta, \eta \vdash \eta^{\prime}$. And we say that it has the MTD whenever: if $\Delta, \eta \vdash \eta^{\prime}$ then $\Delta \vdash \eta \supset \eta^{\prime}$.

For example, modal logics enjoy MTD when considering local reasoning but in general do not have MTD when considering global reasoning. When the logic at hand has both MTMP and MTD we can relate turnstile and arrow interpolation.

Proposition 3.2 Consider a pure propositional based logic where MTD and MTMP hold. Then the logic has turnstile interpolation if and only if it has arrow interpolation. 
Proof: $(\rightarrow)$ Assume that the logic has turnstile interpolation. Let $\vdash \eta \supset \eta^{\prime}$. Then, by MTMP, $\eta \vdash \eta^{\prime}$. Consider three cases:

(a) $\operatorname{var}(\eta) \cap \operatorname{var}\left(\eta^{\prime}\right) \neq \emptyset$. Thus, by Proposition 3.1, there is a formula $\theta$ such that $\operatorname{var}(\theta) \subseteq \operatorname{var}(\eta) \cap \operatorname{var}\left(\eta^{\prime}\right)$ and $\eta \vdash \theta$ and $\theta \vdash \eta^{\prime}$. Hence, by MTD, $\vdash \eta \supset \theta$ and $\vdash \theta \supset \eta^{\prime}$ and so $\theta$ is an arrow interpolant for $\eta$ and $\eta^{\prime}$.

(b) $\operatorname{var}(\eta) \cap \operatorname{var}\left(\eta^{\prime}\right)=\emptyset$ and $\eta \vdash$ ff. Therefore, by MTD, $\vdash \eta \supset$ ff.

(c) $\operatorname{var}(\eta) \cap \operatorname{var}\left(\eta^{\prime}\right)=\emptyset$ and $\vdash \eta^{\prime}$. Immediate.

$(\leftarrow)$ Assume that the logic has arrow interpolation. Let $\Delta \vdash \eta$ and $\Delta^{\prime} \subseteq \Delta$ be a finite set such that $\Delta^{\prime} \vdash \eta$. Then, by MTD,$\vdash \wedge \Delta^{\prime} \supset \eta$. Consider three cases: (a) $\operatorname{var}\left(\Delta^{\prime}\right) \cap \operatorname{var}(\eta) \neq \emptyset$. Thus, there is a formula $\theta$ such that $\operatorname{var}(\theta) \subseteq$ $\operatorname{var}\left(\bigwedge \Delta^{\prime}\right) \cap \operatorname{var}(\eta), \vdash \wedge \Delta^{\prime} \supset \theta$ and $\vdash \theta \supset \eta$. Hence, by MTMP, $\theta \vdash \eta$ and $\wedge \Delta^{\prime} \vdash \theta$ and so $\Delta \vdash \theta$. Therefore, $\theta$ is a turnstile interpolant for $\Delta$ and $\eta$.

(b) $\operatorname{var}\left(\Delta^{\prime}\right) \cap \operatorname{var}(\eta)=\emptyset$ and $\vdash \wedge \Delta^{\prime} \supset$ ff. Then, by MTMP, $\wedge \Delta^{\prime} \vdash$ ff and so $\Delta \vdash \mathrm{ff}$.

(c) $\operatorname{var}\left(\Delta^{\prime}\right) \cap \operatorname{var}(\eta)=\emptyset$ and $\vdash \eta^{\prime}$. Immediate.

QED

\subsection{Interpolation Notions in UCL}

The definition of interpolation in the context of non-pure propositional based logics involves first the choice of the kinds of formulas that can appear as hypotheses and conclusion of relevant derivations. However, the symbols to be considered in the definition of interpolation are always the elements of $X$.

In UCL we only consider turnstile interpolation when the set of hypotheses is the union of a finite set of ambition formulas and a set of formulas possibly with unreliable connectives, and the conclusion is a formula possibly with unreliable connectives. Moreover, in UCL, we only consider arrow interpolation when both the antecedent and the consequent of the implication are formulas possibly with unreliable connectives.

We say that UCL enjoys the turnstile interpolation property for a finite set $\Gamma \subseteq L^{\text {a }}$ and $\Psi \cup\left\{\psi^{\prime}\right\} \subseteq L^{\mathrm{c}}(X)$ with $\Gamma, \Psi \vdash \psi^{\prime}$, if

there is $\Theta \subseteq L^{\mathrm{c}}(X)$ such that

(1) $\operatorname{var}(\Theta) \subseteq \operatorname{var}(\Psi) \cap \operatorname{var}\left(\psi^{\prime}\right)$

(2) $\Gamma, \Psi \vdash^{\text {uc }} \theta$ for each $\theta \in \Theta$ and $\Gamma, \Theta \vdash^{\text {uc }} \psi^{\prime}$

whenever $\operatorname{var}(\Psi) \cap \operatorname{var}\left(\psi^{\prime}\right) \neq \emptyset$,

otherwise either $\Gamma, \Psi \vdash^{\mathrm{uc}}$ ff or $\Gamma \vdash^{\mathrm{uc}} \psi^{\prime}$.

When $\operatorname{var}(\Psi) \cap \operatorname{var}\left(\psi^{\prime}\right) \neq \emptyset$, such a $\Theta$ is said to be a turnstile interpolant for $\Gamma, \Psi$ and $\psi^{\prime}$. UCL has turnstile interpolation if it has turnstile interpolation for every $\Gamma, \Psi$ and $\psi^{\prime}$ such that $\Gamma, \Psi \vdash^{\text {uc }} \psi^{\prime}$.

We say that UCL enjoys the arrow interpolation property for $\psi, \psi^{\prime} \in L^{\mathrm{c}}(X)$ 
with $\vdash^{\text {uc }} \psi \supset \psi^{\prime}$, if

there is $\theta \in L^{\mathrm{c}}(X)$ such that

(1) $\operatorname{var}(\theta) \subseteq \operatorname{var}(\psi) \cap \operatorname{var}\left(\psi^{\prime}\right)$

(2) $\vdash^{\mathrm{uc}} \psi \supset \theta$ and $\vdash^{\mathrm{uc}} \theta \supset \psi^{\prime}$

whenever $\operatorname{var}(\psi) \cap \operatorname{var}\left(\psi^{\prime}\right) \neq \emptyset$,

otherwise either $\vdash^{\mathrm{uc}} \psi \supset \mathrm{ff}$ or $\vdash^{\mathrm{uc}} \psi^{\prime}$.

When $\operatorname{var}(\psi) \cap \operatorname{var}\left(\psi^{\prime}\right) \neq \emptyset$, such a $\theta$ is said to be an arrow interpolant for $\psi$ and $\psi^{\prime}$. UCL has arrow interpolation if it has arrow interpolation for every $\psi$ and $\psi^{\prime}$ such that $\vdash^{\text {uc }} \psi \supset \psi^{\prime}$.

We are ready to discuss the relationship between turnstile and arrow interpolation in the context of UCL along the lines of Subsection 3.1. In the case of $\mathrm{UCL}$, the counterpart of Proposition 3.1 does not hold in general since it is not always the case that if $\Gamma, \Psi \vDash^{\text {uc }} \theta_{1}$ and $\Gamma, \Psi \vDash^{\text {uc }} \theta_{2}$ then $\Gamma, \Psi \vDash^{\text {uc }} \theta_{1} \wedge \theta_{2}$. For example,

$$
\mu \leq \nu^{2} \vDash^{\mathrm{uc}} x_{1} \widetilde{\mathrm{V}}\left(\neg x_{1}\right)
$$

as shown in [32] but

$$
\mu \leq \nu^{2} \not y^{\mathrm{uc}}\left(x_{1} \widetilde{\mathrm{V}}\left(\widetilde{\neg} x_{1}\right)\right) \wedge\left(x_{1} \widetilde{\mathrm{V}}\left(\widetilde{\neg} x_{1}\right)\right) .
$$

Indeed, consider the interpretation $(v, \rho)$ such that $v\left(x_{1}\right)=\perp, \rho(\mu)=0.7$ and $\rho(\nu)=0.9$. Then

- $v \Vdash \delta_{1} \wedge \delta_{1}$;

- $v \Vdash \delta_{2} \wedge \delta_{2}$;

- $v \Vdash \delta_{1} \wedge \delta_{2}$;

- $v \Vdash \delta_{2} \wedge \delta_{1}$

where $\delta_{1}$ is $x_{1} \vee\left(\neg x_{1}\right)$ and $\delta_{2}$ is $x_{1} \nabla\left(\neg x_{1}\right)$. Moreover, $v$ does not satisfy the other possible outcomes of $\left(x_{1} \widetilde{V}\left(\neg x_{1}\right)\right) \wedge\left(x_{1} \widetilde{V}\left(\neg x_{1}\right)\right)$. So

$$
(v, \rho) \Vdash^{\text {uc }} \mu \leq \nu^{2} \text { and }(v, \rho) \mid \nvdash^{\text {uc }}\left(x_{1} \widetilde{V}\left(\widetilde{\neg} x_{1}\right)\right) \wedge\left(x_{1} \widetilde{V}\left(\neg x_{1}\right)\right) .
$$

The relationship between turnstile and arrow interpolations in UCL does not follow so easily, namely because of the restrictions on the MTMP and MTD. We start by discussing MTMP in UCL.

Theorem 3.3 (Metatheorem of modus ponens - MTMP)

Let $\Gamma \subseteq L^{\mathrm{a}}$ be a finite set and $\psi, \psi^{\prime} \in L^{\mathrm{c}}(X)$. Then,

$$
\text { if } \Gamma \vdash^{\mathrm{uc}} \psi \supset \psi^{\prime} \text { then } \Gamma, \psi \vdash^{\mathrm{uc}} \psi^{\prime}
$$

providing that either $\psi$ or $\psi^{\prime}$ is in $L(X)$. 
Proof: The case where $\psi^{\prime} \in L(X)$ follows immediately from MP. We now discuss the case where $\psi^{\prime}$ is not in $L(X)$. Assume that $\psi \in L(X)$ and $\Gamma \vdash^{\text {uc }}$ $\psi \supset \psi^{\prime}$. Consider a derivation of $\psi \supset \psi^{\prime}$ from $\Gamma$ where $\psi \supset \psi^{\prime}$ was obtained using LFT (observe that LFT is the unique rule that can be applied to obtain $\psi \supset \psi^{\prime}$ since $\left.\psi^{\prime} \notin L(X)\right)$. Let

$$
\bigvee_{i=1}^{k}\left(\bigwedge \Phi_{i}\right)
$$

$\Phi_{i} \sqsubseteq_{P_{i}} \psi \supset \psi^{\prime}$ and $\mu \leq P_{i}$, where $\Phi_{i} \subseteq \Omega_{\psi \supset \psi^{\prime}}$, for $i=1, \ldots, k$, be the premises of that LFT application. Then

$$
\vdash \psi \supset \bigvee_{i=1}^{k}\left(\bigwedge\left\{\varphi^{\prime}: \psi \supset \varphi^{\prime} \in \Phi_{i}\right\}\right)
$$

in $\mathrm{PL}$ since

$$
\vdash \bigvee_{i=1}^{k}\left(\bigwedge \Phi_{i}\right)
$$

Hence,

$$
\psi \vdash \bigvee_{i=1}^{k}\left(\bigwedge\left\{\varphi^{\prime}: \psi \supset \varphi^{\prime} \in \Phi_{i}\right\}\right)
$$

Moreover, for each $i=1, \ldots, k$,

$$
\Gamma \vdash^{\mathrm{uc}}\left\{\varphi^{\prime}: \psi \supset \varphi^{\prime} \in \Phi_{i}\right\} \sqsubseteq_{P_{i}} \psi^{\prime}
$$

because $\vdash^{\mathrm{uc}} \Phi_{i} \sqsubseteq_{P_{i}} \psi \supset \psi^{\prime}$ and $\psi \in L(X)$. Therefore by LFT

$$
\Gamma, \psi \vdash^{\mathrm{uc}} \psi^{\prime}
$$

since $\Gamma \vdash^{\mathrm{uc}} \mu \leq P_{i}$ for $i=1, \ldots, k$.

The MTMP only requires restrictions on either the antecedent or the consequent of the target implication.

On the other hand, MTD requires a bit more, namely constraints on the given derivation. For this purpose, one needs two additional notions. Given a derivation $\theta_{1} \ldots \theta_{k}$ of $\psi$ from $\Delta$, we say that $\theta_{i}$ depends on $\delta \in \Delta$ in this derivation if either $\theta_{i}$ is $\delta$ or $\theta_{i}$ is obtained using a rule with at least one of the premises depending on $\delta$. Moreover, an application of MP is said to be classical if both premises are in $L(X)$. The proof of the following result is given in [32].

Theorem 3.4 (Metatheorem of deduction - MTD)

Let $\Gamma \subseteq L^{\mathrm{a}}$ and $\psi, \psi^{\prime} \in L^{\mathrm{c}}(X)$. Assume that $\psi^{\prime}$ fulfills the following proviso: either $\psi^{\prime}$ is distinct from $\psi$ or $\psi^{\prime} \in L(X)$. Then,

$$
\Gamma \vdash^{\mathrm{uc}} \psi \supset \psi^{\prime}
$$


whenever there is a derivation establishing

$$
\Gamma, \psi \vdash^{\mathrm{uc}} \psi^{\prime}
$$

where all the applications of MP over dependents of $\psi$ are classical.

We are now ready to discuss the relationship between turnstile and arrow interpolation in the context of UCL. We start by providing conditions ensuring that arrow interpolation implies turnstile interpolation.

Proposition 3.5 Let $\psi, \psi^{\prime} \in L^{\mathrm{c}}(X)$ such that

- $\psi \vdash^{\mathrm{uc}} \psi^{\prime}$ with a derivation where applications of MP over dependents of $\psi$ are classical;

- UCL enjoys arrow interpolation for $\psi$ and $\psi^{\prime}$ and if $\operatorname{var}(\psi) \cap \operatorname{var}\left(\psi^{\prime}\right) \neq \emptyset$ then the interpolant is in $L(X)$;

- either $\psi^{\prime}$ is distinct from $\psi$ or $\psi^{\prime} \in L(X)$.

Then, UCL enjoys turnstile interpolation for $\psi$ and $\psi^{\prime}$.

Proof: We consider two cases.

(a) $\operatorname{var}(\psi) \cap \operatorname{var}\left(\psi^{\prime}\right) \neq \emptyset$. Observe that, by MTD, $\vdash^{\text {uc }} \psi \supset \psi^{\prime}$. Hence, by arrow interpolation for $\psi$ and $\psi^{\prime}$, there is a formula $\theta$ in $L(X)$ such that

$$
\left\{\begin{array}{l}
\vdash^{\text {uc }} \psi \supset \theta ; \\
\vdash^{\text {uc }} \theta \supset \psi^{\prime} ; \\
\operatorname{var}(\theta) \subseteq \operatorname{var}(\psi) \cap \operatorname{var}\left(\psi^{\prime}\right) .
\end{array}\right.
$$

Then, by MTMP, $\psi \vdash^{\text {uc }} \theta$ and $\theta \vdash^{\text {uc }} \psi^{\prime}$. Therefore, $\theta$ is a turnstile interpolant for $\psi$ and $\psi^{\prime}$.

(b) $\operatorname{var}(\psi) \cap \operatorname{var}\left(\psi^{\prime}\right)=\emptyset$. Observe that, by MTD, $\vdash^{\text {uc }} \psi \supset \psi^{\prime}$. Hence, by arrow interpolation for $\psi$ and $\psi^{\prime}$, either $\vdash^{\mathrm{uc}} \psi \supset \mathrm{ff}$ or $\vdash^{\mathrm{uc}} \psi^{\prime}$. Then, either by MTMP, $\psi \vdash^{\text {uc }} \mathrm{ff}$ or $\vdash^{\text {uc }} \psi^{\prime}$. Therefore, UCL enjoys turnstile interpolation for $\psi$ and $\psi^{\prime}$. QED

We now investigate when a turnstile interpolant is also an arrow interpolant.

Proposition 3.6 Let $\psi$ be a formula in $L^{\mathrm{C}}(X)$ and $\psi^{\prime} \in L(X)$ such that

- $\vdash^{\mathrm{uc}} \psi \supset \psi^{\prime}$;

- $\operatorname{var}(\psi) \cap \operatorname{var}\left(\psi^{\prime}\right) \neq \emptyset$;

- there is a turnstile interpolant $\theta \in L^{\mathrm{c}}(X)$ for $\psi$ and $\psi^{\prime}$ such that

- there is a derivation for $\psi \vdash^{\mathrm{uc}} \theta$ where applications of MP over dependents of $\psi$ are classical; 
- there is a derivation for $\theta \vdash^{\mathrm{uc}} \psi^{\prime}$ where applications of MP over dependents of $\theta$ are classical;

- either $\theta$ is distinct from $\psi$ or $\theta \in L(X)$.

Then, $\theta$ is an arrow interpolant for $\Gamma, \psi$ and $\psi^{\prime}$.

Proof: Observe that $\psi \vdash^{\text {uc }} \psi^{\prime}$ by MTMP. Let $\theta$ be a turnstile interpolant for $\psi$ and $\psi^{\prime}$ in the conditions above. Then by MTD $\vdash^{\text {uc }} \psi \supset \theta$ and $\vdash^{\text {uc }} \theta \supset \psi^{\prime}$. Therefore, $\theta$ is an arrow interpolant for $\psi$ and $\psi^{\prime}$.

QED

We get a result similar to Proposition 3.6 when $\psi \in L(X)$ and the conditions are modified accordingly.

We now provide conditions ensuring that turnstile interpolation implies arrow interpolation when there is no sharing of propositional variables between the relevant formulas for interpolation.

Proposition 3.7 Let $\psi, \psi^{\prime} \in L^{\mathrm{c}}(X)$ such that

- $\vdash^{\mathrm{uc}} \psi \supset \psi^{\prime}$;

- $\operatorname{var}(\psi) \cap \operatorname{var}\left(\psi^{\prime}\right)=\emptyset$;

- UCL enjoys turnstile interpolation for $\psi$ and $\psi^{\prime}$;

- $\psi \in L(X)$.

Then, UCL enjoys arrow interpolation for $\psi$ and $\psi^{\prime}$.

Proof: Observe that, by MTMP, $\psi \vdash^{\text {uc }} \psi^{\prime}$. Hence, by turnstile interpolation for $\psi$ and $\psi^{\prime}$, either $\psi \vdash^{\text {uc }}$ ff or $\vdash^{\text {uc }} \psi^{\prime}$. Consider two cases:

(a) $\psi \vdash^{\text {uc }}$ ff. Then $\psi \vdash$ ff, since $\mathrm{UCL}$ is a conservative extension of PL, see Theorem 2.3 in [32]. Hence, $\vdash \psi \supset \mathrm{ff}$ by MTD. So, $\vdash^{\mathrm{uc}} \psi \supset \mathrm{ff}$ since UCL is an extension of PL, see Theorem 2.3 in [32]. Thus, UCL enjoys arrow interpolation for $\psi$ and $\psi^{\prime}$.

$\vdash^{\text {uc }} \psi^{\prime}$. Then it immediately follows that UCL enjoys arrow interpolation for $\psi$ and $\psi^{\prime}$.

QED

\section{Arrow Interpolation in UCL}

Herein we prove that $\mathrm{UCL}$ enjoys arrow interpolation. We start by showing that, in $\mathrm{UCL}$, each outcome of a valid formula in $L^{\mathrm{c}}(X)$ is a tautology.

Proposition 4.1 Let $\psi$ be a formula in $L^{\mathrm{c}}(X)$ such that $\vDash^{\mathrm{uc}} \psi$. Then $\vDash \varphi$ for every $\varphi \in \Omega_{\psi}$. 
Proof: Let $v$ be a valuation. Since, $\vDash^{\text {uc }} \psi$, then $(v, \rho) \Vdash^{\text {uc }} \psi$ where $\rho$ is an assignment such that $\rho(\mu)=1$ and $\rho(\nu)<1$. Hence

$$
\mathbb{R} \rho \Vdash^{\text {fo }} 1=\sum_{\substack{\varphi \in \Omega_{\psi} \\ v \Vdash \varphi}} \mathfrak{P}[\psi \triangleright \varphi] .
$$

On the other hand,

$$
\mathbb{R} \rho \Vdash^{\text {fo }} 1=\sum_{\varphi \in \Omega_{\psi}} \mathfrak{P}[\psi \triangleright \varphi]
$$

because $\vdash^{\text {uc }} \Omega_{\psi} \sqsubseteq_{1} \psi$. Since, for every $\varphi \in \Omega_{\psi}$,

$$
\mathbb{R} \rho \Vdash^{\text {fo }} \mathfrak{P}[\psi \triangleright \varphi]>0
$$

then, $v \Vdash \varphi$ for every $\varphi \in \Omega_{\psi}$.

Now we show an auxiliary result about propositional satisfaction under particular substitutions. Before we need the following notation. Given $\psi, \eta \in L^{\mathrm{c}}(X)$ and $x \in X$, we denote by $\psi_{\eta}^{x}$ the formula in $L^{\mathrm{c}}(X)$ obtained by simultaneously replacing in $\psi$ the occurrences of variable $x$ by formula $\eta$.

Proposition 4.2 Let $v$ be a valuation, $\varphi \in L(X)$ and $y \in X$. Then

- $v \Vdash \varphi$ iff $v \Vdash \varphi_{\mathrm{t}}^{y}$, whenever $v(y)=\top$;

- $v \Vdash \varphi$ iff $v \Vdash \varphi_{\mathrm{ff}}^{y}$, whenever $v(y)=\perp$.

Proof: The proof follows by a straighforward induction.

We are ready to state the existence of arrow interpolants for formulas sharing propositional variables.

Proposition 4.3 Let $\psi, \psi^{\prime} \in L^{\mathrm{c}}(X)$. Assume that $\vdash^{\mathrm{uc}} \psi \supset \psi^{\prime}$ and $\operatorname{var}(\psi) \cap$ $\operatorname{var}\left(\psi^{\prime}\right) \neq \emptyset$. Then there is an arrow interpolant $\theta \in L(X)$ for $\psi$ and $\psi^{\prime}$.

Proof: Let $\operatorname{var}(\psi) \backslash \operatorname{var}\left(\psi^{\prime}\right)=Y$. We prove the result by induction on the number of elements in $Y$.

Base: $|Y|=0$. Let

$$
\theta=\bigvee_{\varphi \in \Omega_{\psi}} \varphi
$$

We now show that $\theta$ is an interpolant for $\psi$ and $\psi^{\prime}$.

(1) $\vdash^{\text {uc }} \psi \supset \theta$. Indeed,

$$
\vdash \bigwedge_{\varphi \in \Omega_{\psi}}(\varphi \supset \theta)
$$

by propositional reasoning. Moreover,

$$
\vdash^{\text {uc }}\left\{\varphi \supset \theta: \varphi \in \Omega_{\psi}\right\} \sqsubseteq_{1} \psi \supset \theta
$$


and $\vdash^{\mathrm{uc}} \mu \leq 1$. Therefore, by LFT, $\vdash^{\mathrm{uc}} \psi \supset \theta$.

(2) $\vdash^{\text {uc }} \theta \supset \psi^{\prime}$. Observe that, by soundness of $\mathrm{UCL}, \vDash^{\text {uc }} \psi \supset \psi^{\prime}$ and so, by Proposition 4.1, for every $\varphi^{\prime} \in \Omega_{\psi^{\prime}}$,

$$
\vDash \varphi \supset \varphi^{\prime}, \quad \text { for each } \varphi \in \Omega_{\psi} .
$$

Therefore, by propositional reasoning and completeness of PL,

$$
\vdash \bigwedge_{\varphi^{\prime} \in \Omega_{\psi^{\prime}}} \theta \supset \varphi^{\prime} .
$$

Moreover,

$$
\vdash^{\text {uc }}\left\{\theta \supset \varphi^{\prime}: \varphi^{\prime} \in \Omega_{\psi^{\prime}}\right\} \sqsubseteq_{1} \theta \supset \psi^{\prime}
$$

and $\vdash^{\mathrm{uc}} \mu \leq 1$. Therefore, by LFT, $\vdash^{\mathrm{uc}} \theta \supset \psi^{\prime}$.

(3) $\operatorname{var}(\theta) \subseteq \operatorname{var}(\psi) \cap \operatorname{var}\left(\psi^{\prime}\right)$. Observe that $\operatorname{var}(\varphi)=\operatorname{var}(\psi)$ for each $\varphi \in \Omega_{\psi}$ and that

$$
\operatorname{var}(\theta) \subseteq \bigcup_{\varphi \in \Omega_{\psi}} \operatorname{var}(\varphi)=\operatorname{var}(\psi) \subseteq \operatorname{var}\left(\psi^{\prime}\right) .
$$

So $\operatorname{var}(\theta) \subseteq \operatorname{var}(\psi) \cap \operatorname{var}\left(\psi^{\prime}\right)$.

Step: $|Y|=k+1$. Let $y \in Y$. We start by showing that

$$
\vdash^{\mathrm{uc}} \psi_{\mathrm{tt}}^{y} \supset \psi^{\prime} \text { and } \vdash^{\mathrm{uc}} \psi_{\mathrm{ff}}^{y} \supset \psi^{\prime} .
$$

(a) $\vdash^{\text {uc }} \psi_{\mathrm{tt}}^{y} \supset \psi^{\prime}$. Since $\vdash^{\mathrm{uc}} \psi \supset \psi^{\prime}$, by Proposition 4.1, the soundness of UCL and the completeness of PL, we have

$$
\vdash \varphi \supset \varphi^{\prime}, \quad \text { for each } \varphi \in \Omega_{\psi}, \varphi^{\prime} \in \Omega_{\psi^{\prime}}
$$

and so, by closure for substitution in PL,

$$
\vdash \varphi_{\mathrm{t}}^{y} \supset \varphi^{\prime}, \quad \text { for each } \varphi \in \Omega_{\psi}, \varphi^{\prime} \in \Omega_{\psi^{\prime}} .
$$

Since $\varphi_{\mathrm{t}}^{y} \in \Omega_{\psi_{\mathrm{t}}^{y}}$ iff $\varphi \in \Omega_{\psi}$ then

$$
\vdash \bigwedge_{\varphi_{\mathbf{t}}^{y} \in \Omega_{\psi_{\mathbb{t}}^{y}, \varphi^{\prime} \in \Omega_{\psi^{\prime}}}} \varphi_{\mathrm{tt}}^{y} \supset \varphi^{\prime} .
$$

Moreover,

$$
\vdash^{\mathrm{uc}}\left\{\varphi_{\mathrm{tt}}^{y} \supset \varphi^{\prime}: \varphi_{\mathrm{tt}}^{y} \in \Omega_{\psi_{\mathrm{t}}^{y}}, \varphi^{\prime} \in \Omega_{\psi^{\prime}}\right\} \sqsubseteq_{1} \psi_{\mathrm{tt}}^{y} \supset \psi^{\prime}
$$

and $\vdash^{\text {uc }} \mu \leq 1$. Therefore, by LFT, $\vdash^{\text {uc }} \psi_{\mathrm{t}}^{y} \supset \psi^{\prime}$.

(b) $\vdash^{\text {uc }} \psi_{\mathrm{ff}}^{y} \supset \psi^{\prime}$. The proof is similar to (1).

Capitalizing on (a) and (b) we now prove that

$$
\vdash^{\mathrm{uc}}\left(\psi_{\mathrm{tt}}^{y} \vee \psi_{\mathrm{ff}}^{y}\right) \supset \psi^{\prime} .
$$


Indeed,

$$
\vdash^{\text {uc }}\left(\varphi_{\mathrm{tt}}^{y} \vee \varphi_{\mathrm{ff}}^{y}\right) \supset \varphi^{\prime}, \quad \text { for each } \varphi_{\mathrm{tt}}^{y} \in \Omega_{\psi_{\mathrm{t}}^{y}}, \varphi_{\mathrm{ff}}^{y} \in \Omega_{\psi_{\mathrm{f}}^{y}}, \varphi^{\prime} \in \Omega_{\psi^{\prime}}
$$

by Proposition 4.1, (a) and (b). So, by LFT, $\vdash^{\text {uc }}\left(\psi_{\mathrm{t}}^{y} \vee \psi_{\mathrm{ff}}^{y}\right) \supset \psi^{\prime}$ since

$$
\vdash^{\mathrm{uc}}\left\{\left(\varphi_{\mathrm{tt}}^{y} \vee \varphi_{\mathrm{ff}}^{y}\right) \supset \varphi^{\prime}: \varphi_{\mathrm{tt}}^{y} \in \Omega_{\psi_{\mathrm{t}}^{y}}, \varphi_{\mathrm{ff}}^{y} \in \Omega_{\psi_{\mathrm{f}}^{y}}, \varphi^{\prime} \in \Omega_{\psi^{\prime}}\right\} \sqsubseteq_{1}\left(\psi_{\mathrm{t}}^{y} \vee \psi_{\mathrm{ff}}^{y}\right) \supset \psi^{\prime}
$$

and $\vdash^{\mathrm{uc}} \mu \leq 1$.

Using the induction hypothesis over $\vdash^{\mathrm{uc}}\left(\psi_{\mathrm{t}}^{y} \vee \psi_{\mathrm{ff}}^{y}\right) \supset \psi^{\prime}$ let $\theta \in L(X)$ be an arrow interpolant for $\psi_{\mathrm{tt}}^{y} \vee \psi_{\mathrm{ff}}^{y}$ and $\psi^{\prime}$. We now show that $\theta$ is also an arrow interpolant for $\psi$ and $\psi^{\prime}$.

(1) $\vdash^{\text {uc }} \psi \supset \theta$. We start by proving that

$$
(*) \vDash \varphi \supset \theta, \quad \text { for every } \varphi \in \Omega_{\psi} .
$$

Indeed, let $v$ be a valuation and $\varphi \in \Omega_{\psi}$ such that $v \Vdash \varphi$. Then, by Proposition 4.2 ,

$$
v \Vdash \varphi_{\mathrm{tt}}^{y} \vee \varphi_{\mathrm{ff}}^{y} .
$$

On the other hand, since $\vdash^{\text {uc }}\left(\psi_{\mathrm{t}}^{y} \vee \psi_{\mathrm{ff}}^{y}\right) \supset \theta$, then, by soundness of UCL and Proposition 4.1, we have $v \Vdash \theta$.

So, by $(*)$, completeness of $\mathrm{PL}$, the fact that $\mathrm{UCL}$ is an extension of $\mathrm{PL}$, and by LFT, we have $\vdash^{\text {uc }} \psi \supset \theta$.

(2) $\vdash^{\text {uc }} \theta \supset \psi^{\prime}$ comes from the fact that $\theta$ is an arrow interpolant for $\psi_{\mathrm{tt}}^{y} \vee \psi_{\mathrm{ff}}^{y}$ and $\psi^{\prime}$.

$(3) \operatorname{var}(\theta) \subseteq \operatorname{var}(\psi) \cap \operatorname{var}\left(\psi^{\prime}\right)$. Since $\operatorname{var}(\theta) \subseteq \operatorname{var}\left(\psi_{\mathrm{t}}^{y} \vee \psi_{\mathrm{ff}}^{y}\right) \cap \operatorname{var}\left(\psi^{\prime}\right) \subseteq \operatorname{var}(\psi) \cap$ $\operatorname{var}\left(\psi^{\prime}\right)$ the thesis follows.

QED

Observe that the construction proposed in the proof of the preceding proposition allows to obtain an arrow uniform interpolant since it depends only on the antecedent and on the common language between the antecedent and the consequent.

Theorem 4.4 UCL has arrow interpolation.

Proof: Assume that $\vdash^{\text {uc }} \psi \supset \psi^{\prime}$. Consider two cases:

(a) $\operatorname{var}(\psi) \cap \operatorname{var}\left(\psi^{\prime}\right) \neq \emptyset$. Then the thesis follows by Proposition 4.3.

(b) $\operatorname{var}(\psi) \cap \operatorname{var}\left(\psi^{\prime}\right)=\emptyset$. By soundness of UCL, $\vDash^{\text {uc }} \psi \supset \psi^{\prime}$. Then, by Proposition 4.1, $\vDash \varphi \supset \varphi^{\prime}$ for every $\varphi \in \Omega_{\psi}$ and $\varphi^{\prime} \in \Omega_{\psi^{\prime}}$. So, by interpolation in PL, either $\vdash \varphi \supset \mathrm{ff}$ or $\vdash \varphi^{\prime}$ for every $\varphi \in \Omega_{\psi}$ and $\varphi^{\prime} \in \Omega_{\psi^{\prime}}$. Consider two cases:

(i) $\vdash \varphi \supset$ ff for each $\varphi \in \Omega_{\psi}$. Then by LFT, $\vdash^{\text {uc }} \psi \supset$ ff.

(ii) $\forall \varphi \supset$ ff for some $\varphi \in \Omega_{\psi}$. Then $\vdash \varphi^{\prime}$ for every $\varphi^{\prime} \in \Omega_{\psi^{\prime}}$ since $\vdash \varphi \supset \varphi^{\prime}$ for every $\varphi^{\prime} \in \Omega_{\psi^{\prime}}$. Then by LFT, $\vdash^{\text {uc }} \psi^{\prime}$.

QED 


\section{Turnstile Interpolation in UCL}

In order to investigate turnstile interpolation in UCL, we need to prove first some technical results. For this purpose we introduce a particular kind of substitution so that we can reduce derivations in UCL to derivations in PL under some conditions.

An outcome substitution is a map $\alpha: \widetilde{\Sigma} \rightarrow \Sigma$ such that $\alpha(\widetilde{c})$ is either $c$ or $\bar{c}$, for each $\widetilde{c} \in \widetilde{\Sigma}$. This map can be easily extended to formulas in $L^{\mathrm{c}}(X)$ as well as to sets of formulas. Given a formula $\psi$ in $L^{\mathrm{c}}(X)$, we use $\alpha(\psi)$ to denote the formula in $L(X)$ resulting from applying $\alpha$ to $\psi$. Similarly, we use $\alpha(\Psi)$ when $\Psi$ is a set of formulas in $L^{\mathrm{c}}(X)$.

The first result concerns reflection of derivations from UCL to PL by an outcome substitution.

Proposition 5.1 Let $\Gamma \subseteq L^{\mathrm{a}}, \Psi \cup\left\{\psi^{\prime}\right\} \subseteq L^{\mathrm{c}}(X)$ and $\alpha$ an outcome substitution. Assume that $\Gamma, \Psi \vdash^{\text {uc }} \psi^{\prime}$ with a derivation not using the LFT rule. Then $\alpha(\Psi) \vdash$ $\alpha\left(\psi^{\prime}\right)$.

Proof: The proof follows by induction on the length of a derivation of $\Gamma, \Psi \vdash^{\text {uc }} \psi^{\prime}$ not using LFT.

Base:

(1) $\psi^{\prime}$ is justified by TAUT. Then $\psi^{\prime} \in L(X)$ and $\vDash \psi^{\prime}$. Hence, by completeness of PL and monotonicity, $\alpha(\Psi) \vdash \alpha\left(\psi^{\prime}\right)$ since $\alpha\left(\psi^{\prime}\right)=\psi^{\prime}$;

(2) $\psi^{\prime}$ is in $\Psi$. Then $\alpha\left(\psi^{\prime}\right)$ is in $\alpha(\Psi)$.

Step: $\psi^{\prime}$ is obtained by MP from $\psi^{\prime \prime}$ and $\psi^{\prime \prime} \supset \psi^{\prime}$. By the induction hypothesis, $\alpha(\Psi) \vdash \alpha\left(\psi^{\prime \prime}\right)$ and $\alpha(\Psi) \vdash \alpha\left(\psi^{\prime \prime}\right) \supset \psi^{\prime}$. So, again by MP, the thesis follows. QED

The following result shows that derivations in PL of all outcomes of a formula from each outcome of a single hypothesis lead to a derivation in UCL.

Proposition 5.2 Let $\psi, \psi^{\prime} \in L^{\mathrm{C}}(X)$. Assume that, $\varphi \vdash \varphi^{\prime}$ for every $\varphi \in \Omega_{\psi}$ and $\varphi^{\prime} \in \Omega_{\psi^{\prime}}$. Then $\psi \vdash^{\mathrm{uc}} \psi^{\prime}$.

Proof: Assume that, $\varphi \vdash \varphi^{\prime}$ for every $\varphi \in \Omega_{\psi}$ and $\varphi^{\prime} \in \Omega_{\psi^{\prime}}$. Then by MTD in PL,

$$
\vdash \varphi \supset \varphi^{\prime}
$$

for each $\varphi \in \Omega_{\psi}$ and $\varphi^{\prime} \in \Omega_{\psi^{\prime}}$. Hence

$$
\vdash \bigwedge_{\varphi \in \Omega_{\psi}}\left(\varphi \supset \varphi^{\prime}\right)
$$

for every $\varphi^{\prime} \in \Omega_{\psi^{\prime}}$. Therefore, by LFT, $\vdash^{\text {uc }} \psi \supset \varphi^{\prime}$ for every $\varphi^{\prime} \in \Omega_{\psi^{\prime}}$. Thus, by MTMP in UCL (see Theorem 3.3), $\psi \vdash^{\mathrm{uc}} \varphi^{\prime}$ for every $\varphi^{\prime} \in \Omega_{\psi^{\prime}}$. Finally, by LFT, $\psi \vdash^{\text {uc }} \psi^{\prime}$.

QED 
The next result states that hypotheses in $L^{\mathrm{c}}(X)$ play no role when deriving outcome and ambition formulas.

Proposition 5.3 Let $\Gamma \subseteq L^{\mathrm{a}}$ be a finite set, $\Psi \subseteq L^{\mathrm{c}}(X)$ and $\eta \in L^{\mathrm{a}} \cup L^{\circ}(X)$. Assume that $\Gamma, \Psi \vdash^{\mathrm{uc}} \eta$. Then $\Gamma \vdash^{\mathrm{uc}} \eta$.

Proof: Consider two cases:

(1) $\eta \in L^{\circ}(X)$. The proof follows by induction on the length of a derivation for $\Gamma, \Psi \vdash^{\text {uc }} \eta$.

Base: $\eta$ is justified either by NO or by SO. Then $\vdash^{\mathrm{uc}} \eta$ and so, by monotonicity, $\Gamma \vdash^{\text {uc }} \eta$.

Step: $\eta$ is justified either by AO or by WO. Assume that $\eta$ is $\Phi \sqsubseteq_{P_{2}} \psi$ and was obtained from $\Phi \sqsubseteq_{P_{1}} \psi$ by WO. Then, by the induction hypothesis, $\Gamma \vdash^{\text {uc }}$ $\Phi \sqsubseteq_{P_{1}} \psi$ and so, once again by WO, we conclude that $\Gamma \vdash^{\mathrm{uc}} \Phi \sqsubseteq_{P_{2}} \psi$. Similarly, when $\eta$ is justified by AO.

(2) $\eta \in L^{\mathrm{a}}$. In this case $\eta$ is obtained by WA from the premises $\eta_{1}, \ldots, \eta_{k}$ in $L^{\mathrm{a}}$. Then, by the induction hypothesis, $\Gamma \vdash^{\text {uc }} \eta_{i}$ for each $i=1, \ldots, k$ and so, again by rule WA, we can conclude that $\Gamma \vdash^{\mathrm{uc}} \eta$.

QED

The next result states the existence of turnstile interpolants under certain conditions.

Proposition 5.4 Let $\Gamma \subseteq L^{\text {a }}$ be a finite set and $\psi, \psi^{\prime} \in L^{\mathrm{c}}(X)$ where in $\psi$ there is at most one occurrence of each unreliable connective. Assume that

- $\operatorname{var}(\psi) \cap \operatorname{var}\left(\psi^{\prime}\right) \neq \emptyset$;

- $\Gamma, \psi \vdash^{\mathrm{uc}} \psi^{\prime}$ with a derivation having formulas $\varphi_{1}, \ldots, \varphi_{n} \in L\left(\operatorname{var}\left(\psi^{\prime}\right)\right)$ such that $\left\{\varphi_{1}, \ldots, \varphi_{n}\right\} \vdash^{\mathrm{uc}} \psi^{\prime}$ and no LFT rule was applied in the subderivations of $\varphi_{1}, \ldots, \varphi_{n}$.

Then there is a turnstile interpolant for $\Gamma, \psi$ and $\psi^{\prime}$.

Proof: Consider a derivation of $\Gamma, \psi \vdash^{\text {uc }} \psi^{\prime}$ having formulas $\varphi_{1}, \ldots, \varphi_{n} \in$ $L\left(\operatorname{var}\left(\psi^{\prime}\right)\right)$ such that $\left\{\varphi_{1}, \ldots, \varphi_{n}\right\} \vdash^{\text {uc }} \psi^{\prime}$ and no LFT rule was applied in the subderivations of $\varphi_{1}, \ldots, \varphi_{n}$. Hence

$$
\Gamma, \psi \vdash^{\mathrm{uc}} \bigwedge_{i=1}^{n} \varphi_{i}
$$

and so by Proposition 5.1,

$$
\alpha(\psi) \vdash \bigwedge_{i=1}^{n} \varphi_{i}
$$

for each outcome substitution $\alpha$. Then, by PL interpolation, for each outcome substitution $\alpha$, there is a turnstile interpolant $\theta_{\alpha}$ such that

$$
\text { - } \operatorname{var}\left(\theta_{\alpha}\right) \subseteq \operatorname{var}(\alpha(\psi)) \cap \operatorname{var}\left(\bigwedge_{i=1}^{n} \varphi_{i}\right) ;
$$


- $\alpha(\psi) \vdash \theta_{\alpha}$ and $\theta_{\alpha} \vdash \bigwedge_{i=1}^{n} \varphi_{i}$.

Let

$$
\theta \text { be } \bigvee_{\alpha} \theta_{\alpha}
$$

Then

$$
\varphi \vdash \theta
$$

for each $\varphi \in \Omega_{\psi}$ since

$$
\Omega_{\psi}=\{\alpha(\psi): \alpha \text { is an outcome substitution }\} .
$$

Hence, by Proposition 5.2 and monotonicity,

$$
\text { (1) } \Gamma, \psi \vdash^{\mathrm{uc}} \theta \text {. }
$$

Moreover,

$$
\theta \vdash \bigwedge_{i=1}^{n} \varphi_{i}
$$

and so

$$
\theta \vdash \varphi_{i} \text {, for each } i=1, \ldots, n \text {. }
$$

Thus,

$$
\theta \vdash^{\mathrm{uc}} \varphi_{i} \text {, for each } i=1, \ldots, n
$$

and so

$$
\text { (2) } \theta \vdash^{\text {uc }} \psi^{\prime}
$$

since $\left\{\varphi_{1}, \ldots, \varphi_{n}\right\} \vdash^{\text {uc }} \psi^{\prime}$. Furthermore,

(3) $\operatorname{var}(\theta)=\bigcup_{\alpha} \operatorname{var}\left(\theta_{\alpha}\right) \subseteq \bigcup_{\alpha} \operatorname{var}(\alpha(\psi)) \cap \operatorname{var}\left(\bigwedge_{i=1}^{n} \varphi_{i}\right) \subseteq \operatorname{var}(\psi) \cap \operatorname{var}\left(\psi^{\prime}\right)$.

So, by (1), (2) and (3), $\theta$ is a turnstile interpolant for $\Gamma, \psi$ and $\psi^{\prime}$. QED

The following result states the conditions that guarantee that UCL has turnstile interpolation.

Theorem 5.5 Let $\Gamma \subseteq L^{\mathrm{a}}$ be a finite set and $\psi, \psi^{\prime} \in L^{\mathrm{c}}(X)$ such that $\Gamma, \psi \vdash^{\mathrm{uc}} \psi^{\prime}$ with a derivation having formulas $\varphi_{1}, \ldots, \varphi_{n} \in L\left(\operatorname{var}\left(\psi^{\prime}\right)\right)$ such that $\left\{\varphi_{1}, \ldots, \varphi_{n}\right\} \vdash^{\text {uc }}$ $\psi^{\prime}$. Then, UCL has turnstile interpolation for $\Gamma, \psi$ and $\psi^{\prime}$ whenever:

- if $\operatorname{var}(\psi) \cap \operatorname{var}\left(\psi^{\prime}\right) \neq \emptyset$ then each unreliable connective occurs at most once in $\psi$ and the LFT rule was not applied in the subderivations of $\varphi_{1}, \ldots, \varphi_{n}$;

- if $\operatorname{var}(\psi) \cap \operatorname{var}\left(\psi^{\prime}\right)=\emptyset$ then $\psi$ is in $L(X)$, each unreliable connective occurs at most once in $\psi^{\prime}$. 
Proof: Assume that $\Gamma, \psi \vdash^{\text {uc }} \psi^{\prime}$. Consider two cases:

(a) $\operatorname{var}(\psi) \cap \operatorname{var}\left(\psi^{\prime}\right) \neq \emptyset$. Then the thesis follows by Proposition 5.4.

(b) $\operatorname{var}(\psi) \cap \operatorname{var}\left(\psi^{\prime}\right)=\emptyset$. Observe that

$$
\Gamma, \psi \vdash^{\mathrm{uc}} \bigwedge_{i=1}^{n} \varphi_{i} .
$$

Then, by Proposition 5.1,

$$
\psi \vdash \bigwedge_{i=1}^{n} \varphi_{i} .
$$

So, by interpolation in PL, either $\psi \vdash \mathrm{ff}$ or $\vdash \bigwedge_{i=1}^{n} \varphi_{i}$. Hence, the thesis follows straightforwardly.

QED

We now provide an illustration of a turnstile interpolant for $\mu \leq \nu, x_{2} \supset x_{1}$ and $\left(\left(\neg x_{1}\right) \supset\left(\neg x_{2}\right)\right) \vee\left(\neg x_{3}\right)$. Observe that

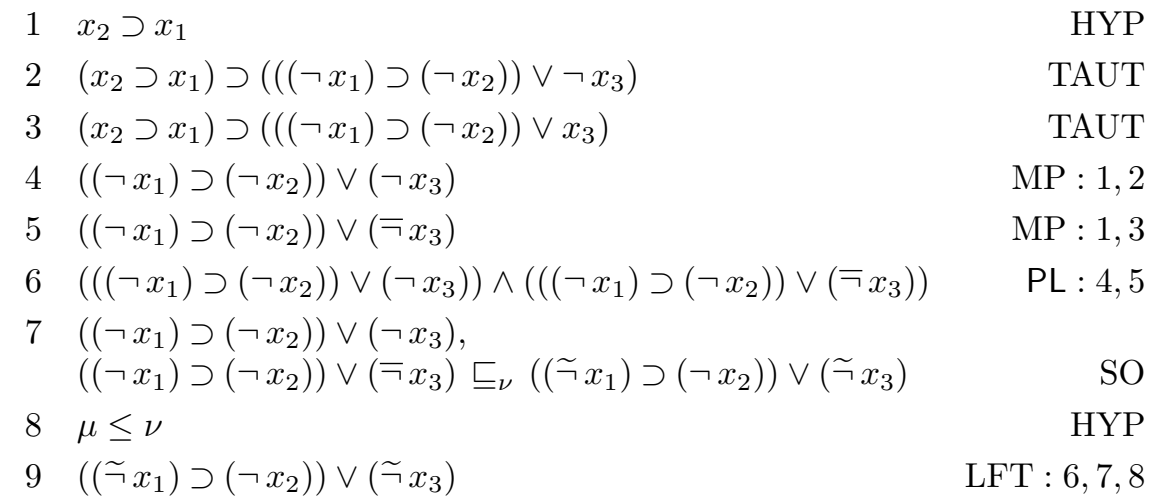

Figure 1: $\mu \leq \nu, x_{2} \supset x_{1} \vdash^{\text {uc }}\left(\left(\neg x_{1}\right) \supset\left(\neg x_{2}\right)\right) \vee\left(\neg x_{3}\right)$.

$$
\mu \leq \nu, x_{2} \supset x_{1} \vdash^{\mathrm{uc}}\left(\left(\neg x_{1}\right) \supset\left(\neg x_{2}\right)\right) \vee\left(\neg x_{3}\right)
$$

as can be shown in Figure 1, and that the conditions of Proposition 5.4 are satisfied. Consider an outcome substitution $\alpha$ such that $\alpha(\widetilde{\neg})=\neg$. Then

$$
x_{2} \supset x_{1} \vdash\left(\left(\left(\neg x_{1}\right) \supset\left(\neg x_{2}\right)\right) \vee\left(\neg x_{3}\right)\right) \wedge\left(\left(\left(\neg x_{1}\right) \supset\left(\neg x_{2}\right)\right) \vee\left(\neg x_{3}\right)\right)
$$

which has $\left(\neg x_{1}\right) \supset\left(\neg x_{2}\right)$ as an interpolant. Similarly, for the outcome substitution $\alpha$ such that $\alpha(\widetilde{\neg})=\bar{\neg}$. Thus, according to the proof of that proposition, $\left(\neg x_{1}\right) \supset\left(\neg x_{2}\right)$ is an interpolant for $\mu \leq \nu, x_{2} \supset x_{1}$ and $\left(\left(\neg x_{1}\right) \supset\left(\neg x_{2}\right)\right) \vee\left(\neg x_{3}\right)$. 


\section{Outlook}

Both arrow and turnstile interpolation were investigated in the context of $\mathrm{UCL}$, (cf [32]), a complete extension of classical propositional logic for reasoning about unreliable connectives that behave correctly with a given probability. The first step was the fine tuning of the notions of interpolation in order to cope with the different kinds of formulas present in UCL. Then, we were able to prove that UCL has unrestricted arrow interpolation. Furthermore, we also showed that UCL enjoys turnstile interpolation under some moderate provisos. These provisos came as no surprise because, among other reasons, in $\mathrm{UCL}$ the rule of conjunction introduction is not sound, validity is not always preserved by substitution, and the metatheorems of deduction and modus ponens both require provisos.

Concerning future work, we plan to assess the practical impact of the interpolation properties of UCL when applying this logic to the verification of circuits with unreliable gates. Like in other application scenarios, we expect to find better algorithms by making good use of interpolants.

On another front, we intend to extend UCL in order to cope with probabilistic propositional variables and analyze its properties namely interpolation. It seems also interesting to extend this probabilistic setting to propositional logics with modal-like operators, as well as to quantification logics.

\section{Acknowledgments}

The authors are grateful to the referees who suggested several improvements to the paper. This work was partially supported, under the MCL (MeetCombination of Logics) and PQDR (Probabilistic, Quantum and Differential Reasoning) initiatives of SQIG at IT, by FCT and EU FEDER, namely via the PEst-OE/EEI/LA0008/2013 and AMDSC UTAustin/MAT/0057/2008 projects, as well as by the European Union's Seventh Framework Programme for Research (FP7), namely through project LANDAUER (GA 318287).

\section{References}

[1] C. Areces, P. Blackburn, and M. Marx. Repairing the interpolation theorem in quantified modal logic. Annals of Pure and Applied Logic, 124(1-3):287299, 2003.

[2] C. Areces, E. Hoogland, and D. de Jongh. Interpolation, definability and fixed points in interpretability logics. In Advances in Modal Logic, volume 119 of CSLI Lecture Notes, pages 35-58. CSLI Publications, 2001.

[3] L. D. Beklemishev. On the Craig interpolation and the fixed point properties for GLP. In Proofs, Categories and Computations, volume 13 of Tributes, pages 49-60. College Publications, London, 2010. 
[4] E. W. Beth. On Padoa's method in the theory of definition. Nederl. Akad. Wetensch. Proc. Ser. A. 56 (Indagationes Math.), 15:330-339, 1953.

[5] J. Bicarregui, T. Dimitrakos, D. Gabbay, and T. Maibaum. Interpolation in practical formal development. Logic Journal of the IGPL, 9(2):231-243, 2001.

[6] M. P. Bonacina and M. Johansson. On interpolation in decision procedures. In Automated Reasoning with Analytic Tableaux and Related Methods, volume 6793 of Lecture Notes in Computer Science, pages 1-16. Springer, 2011.

[7] A. Carbone. The Craig interpolation theorem for schematic systems. In Collegium Logicum, Vol. 2, volume 2 of Coll. Logicum Ann. Kurt-GödelSoc., pages 87-100. Springer, 1996.

[8] A. Carbone. Interpolants, cut elimination and flow graphs for the propositional calculus. Annals of Pure and Applied Logic, 83(3):249-299, 1997.

[9] W. Carnielli, J. Rasga, and C. Sernadas. Interpolation via translations. Mathematical Logic Quarterly, 55(5):515-534, 2009.

[10] W. A. Carnielli, J. Rasga, and C. Sernadas. Preservation of interpolation features by fibring. Journal of Logic and Computation, 18(1):123-151, 2008.

[11] W. Craig. Linear reasoning. A new form of the Herbrand-Gentzen theorem. The Journal of Symbolic Logic, 22:250-268, 1957.

[12] W. Craig. Three uses of the Herbrand-Gentzen theorem in relating model theory and proof theory. The Journal of Symbolic Logic, 22:269-285, 1957.

[13] J. Czelakowski. Sentential logics and Maehara interpolation property. Studia Logica, 44(3):265-283, 1985.

[14] J. Czelakowski and D. Pigozzi. Amalgamation and interpolation in abstract algebraic logic. In Models, Algebras, and Proofs, volume 203 of Lecture Notes in Pure and Applied Mathematics, pages 187-265. Dekker, 1999.

[15] G. D'Agostino. Interpolation in non-classical logics. Synthese. An International Journal for Epistemology, Methodology and Philosophy of Science, 164(3):421-435, 2008.

[16] R. Diaconescu. An institution-independent proof of Craig interpolation theorem. Studia Logica, 77(1):59-79, 2004.

[17] S. Feferman. Harmonious logic: Craig's interpolation theorem and its descendants. Synthese, 164(3):341-357, 2008.

[18] J. M. Font, R. Jansana, and D. Pigozzi. A survey of abstract algebraic logic. Studia Logica, 74(1-2):13-97, 2003. 
[19] D. M. Gabbay and L. Maksimova. Interpolation and Definability, volume 46 of Oxford Logic Guides. The Clarendon Press, Oxford University Press, 2005. Modal and Intuitionistic Logics.

[20] R. Jhala and K. L. McMillan. Interpolant-based transition relation approximation. Logical Methods in Computer Science, 3(4):4:1, 17 pp. (electronic), 2007.

[21] H. J. Keisler and J. M. Keisler. Craig interpolation for networks of sentences. Annals of Pure and Applied Logic, 163(9):1322-1344, 2012.

[22] H. Kihara and H. Ono. Interpolation properties, Beth definability properties and amalgamation properties for substructural logics. Journal of Logic and Computation, 20(4):823-875, 2010.

[23] M. Kracht and F. Wolter. Properties of independently axiomatizable bimodal logics. The Journal of Symbolic Logic, 56(4):1469-1485, 1991.

[24] R. C. Lyndon. An interpolation theorem in the predicate calculus. Pacific Journal of Mathematics, 9:129-142, 1959.

[25] S. Maehara. On the interpolation theorem of Craig. Mathematical Society of Japan. Sūgaku, 12:235-237, 1960/1961.

[26] E. Marchioni and G. Metcalfe. Craig interpolation for semilinear substructural logics. Mathematical Logic Quarterly, 58(6):468-481, 2012.

[27] D. Marker. Model Theory, volume 217 of Graduate Texts in Mathematics. Springer-Verlag, 2002. An Introduction.

[28] K. L. McMillan. An interpolating theorem prover. Theoretical Computer Science, 345(1):101-121, 2005.

[29] C. Miller, S. Kupferschmid, M. Lewis, and B. Becker. Encoding techniques, Craig interpolants and bounded model checking for incomplete designs. In Theory and Applications of Satisfiability Testing-SAT, volume 6175 of Lecture Notes in Computer Science, pages 194-208. Springer, 2010.

[30] F. Montagna. $\Delta$-core fuzzy logics with propositional quantifiers, quantifier elimination and uniform Craig interpolation. Studia Logica, 100(1-2):289317, 2012.

[31] G. F. Schumm. Some failures of interpolation in modal logic. Notre Dame Journal of Formal Logic, 27(1):108-110, 1986.

[32] A. Sernadas, J. Rasga, C. Sernadas, and P. Mateus. Approximate reasoning about logic circuits with single-fan-out unreliable gates. Journal of Logic and Computation, in print.

[33] A. Sernadas, C. Sernadas, and J. Rasga. On meet-combination of logics. Journal of Logic and Computation, 22(6):1453-1470, 2012. 
[34] C. Sernadas, J. Rasga, and A. Sernadas. Preservation of Craig interpolation by the product of matrix logics. Journal of Applied Logic, 11(3):328-349, 2013.

[35] A. Tarski. A Decision Method for Elementary Algebra and Geometry. University of California Press, 1951. 2nd edition.

[36] T. Teige and M. Fränzle. Generalized Craig interpolation for stochastic Boolean satisfiability problems with applications to probabilistic state reachability and region stability. Logical Methods in Computer Science, $8(2): 2: 16,32,2012$.

[37] A. Urquhart. Failure of interpolation in relevant logics. Journal of Philosophical Logic, 22(5):449-479, 1993. 